\title{
Is there life inside black holes?
}

\author{
V I Dokuchaev \\ Institute for Nuclear Research of the Russian Academy of Sciences \\ 60th October Anniversary Prospect 7a, 117312 Moscow, Russia \\ E-mail: dokuchaev@inr.ac.ru
}

\begin{abstract}
Bound inside rotating or charged black holes, there are stable periodic planetary orbits, which neither come out nor terminate at the central singularity. Stable periodic orbits inside black holes exist even for photons. These bound orbits may be defined as orbits of the third kind, following the Chandrasekhar classification of particle orbits in the black hole gravitational field. The existence domain for the third kind orbits is rather spacious, and thus there is place for life inside supermassive black holes in the galactic nuclei. Interiors of the supermassive black holes may be inhabited by civilizations, being invisible from the outside. In principle, one can get information from the interiors of black holes by observing their white hole counterparts.
\end{abstract}

PACS numbers: 04.20.Dw, 04.70.Bw, 96.55.+z, 98.35.Jk, 98.62.Js 


\section{Introduction}

A voyage inside the Black Hole (BH) event horizon may be not finished in the central singularity after a finite proper time of the traveler, but once again outside the event horizon. However, due to the complicated internal BH geometry [1, 2, 3, 4, 5, 6, 7], it would be an emergence in the other universe rather than a return to the traveler's native one. Is it possible to live long inside the $\mathrm{BH}$ avoiding both the downfall to the central singularity and escaping to another universe? To clarify this possibility we suppose that $\mathrm{BH}$ interiors are described by the Kerr-Newman metric with a maximally extended global geometry [1, 2, 3, 4, 8, 9, 10]. These BHs are named the eternal ones. The corresponding Carter-Penrose conformal diagram of the eternal black is an infinite stairway of asymptotically flat space-times, connected by the one-way Einstein-Rosen bridges. The entries and outlets of these Einstein-Rosen bridges are, respectively, the event horizons of the black and white holes. We demonstrate below that living inside the eternal BHs is possible in principle, if these BHs are rotating or charged and massive enough for weakening the tidal forces and radiation of gravitational waves to acceptable level.

A tidal acceleration experienced by the object (e.g., some creature) with a linear size $h$ at a distance $r$ from a black hole with mass $M$ is of the order of $g_{t} \sim G M h / r^{3}$ (see e.g. Chapter 32.6 in [1]). Let us accept that this tidal acceleration $g_{t}$ is in a comfortable range for living species, if it does not exceed the corresponding gravitational acceleration on the surface of the Earth, $g_{\mathrm{E}} \simeq 10 \mathrm{~m} / \mathrm{sec}^{2}$. From equality $g_{t}=g_{\mathrm{E}}$ we find the corresponding tidal radius $r_{t} \sim\left(M G h / g_{\mathrm{E}}\right)^{1 / 3}$. For a comfortable traveling inside black hole this tidal radius must be less than the black hole event horizon radius, $r_{t}<r_{+} \simeq G M / c^{2}$. From this inequality we estimate a corresponding minimal black hole mass:

$$
M>M_{\min } \sim \frac{h^{1 / 2} c^{3}}{g_{\mathrm{E}}^{1 / 2} G} \simeq 6.510^{3} \sqrt{h} M_{\odot} .
$$

The minimal black hole mass $M_{\min }$ in (11) is much more less than masses of the supermassive black hole candidates in the galactic nuclei, $M \sim 10^{6}-10^{10} M_{\odot}$, for the living species with a linear size $h \sim 1-10^{2} \mathrm{~cm}$.

After traversing the $\mathrm{BH}$ event horizon at radius $r=r_{+}$, a traveler will appear in the $T$-region [11, where his radial coordinate $r$ would become a temporal one and inevitably diminishing towards the central singularity. The irresistible infall in the $T$-region will finish soon after traversing the inner Cauchy horizon at $r=r_{-}<r_{+}$, which is nonzero for the rotating or charged BH. The internal space-time domain $0<r<r_{-}$between the central singularity and the inner $\mathrm{BH}$ horizon is the $R$-region, where stationary observers may exist just as anywhere on the planet Earth. This internal BH domain, hidden by the two horizons from the whole external universe, is indeed a suitable place for safe inhabitation. The only thing needed is to put your vehicle or your planet to a stable periodic orbit inside BH. We discuss some specific properties of stable periodic orbits of planets and photons inside the rotating charged $\mathrm{BH}$, described by the Kerr- 
Newman metric. It is supposed that generic properties of the Kerr-Newman metric are survived inside black holes in spite of a threat from the perturbative instabilities [12, 13, 14, 15, 16, 17].

\section{Stable periodic orbits inside $\mathrm{BH}$}

The most generic description for the motion of particles in the gravitational field of the black holes is a test particle approximation. This approximation is self-consistent, if particles are small and light enough with respect to, respectively, the characteristic size and mass of the black hole, so that the back reaction can be neglected. The term "test planets" is used throughout the paper as a synonym of the "test particles".

Subramanyan Chandrasekhar [2] designated two general types of test particle orbits in the $\mathrm{BH}$ gravitational field: orbits of the first kind, which are completely confined outside the $\mathrm{BH}$ event horizon, and orbits of the second kind, which penetrate inside the $\mathrm{BH}$. Here we also propose to distinguish orbits of a third kind, which are completely bound inside the BH, not escaping outside, nor infalling into the central singularity. The bound orbits of the third kind inside the inner BH horizon were found by Jiří Bičák, Zdeněk Stuchlík and Vladimír Balek [18, 19] for charged particles around the rotating charged BHs (see also [20, 21]) and by Eva Hackmann, Claus Lämmerzahl, Valeria

Kagramanova and Jutta Kunz [22] for neutral particles around rotating BHs (see also [23]). The bound orbits of the third kind are periodic and stable if gravitational and electromagnetic radiation is neglected.

Geodesics equations for neutral test particles and photons and equations of motion for charged particles in the Kerr-Newman metric were derived by B. Carter [10]. According to these equations the motion of test particle with a mass $\mu$ and electric charge $\epsilon$ in the background gravitational field of a $\mathrm{BH}$ with a mass $M$, angular momentum $J=M a$ and electric charge $e$ is completely defined by three integrals of motion: the total particle energy $E$, the azimuthal component of the angular momentum $L$ and the Carter constant $Q$, related with a total angular momentum of the particle. The Carter constant is zero, $Q=0$, if trajectories are confined in the $\mathrm{BH}$ equatorial plane. In particular, the total angular momentum of the particle is $\sqrt{Q+L^{2}}$ in the case of nonrotating $\mathrm{BH}$.

An orbital trajectory of test planet is governed in the Boyer-Lindquist coordinates $(t, r, \theta, \varphi)$ by equations of motion [10, 24]:

$$
\begin{aligned}
& \Sigma \frac{d r}{d \lambda}= \pm \sqrt{V_{r}}, \\
& \Sigma \frac{d \theta}{d \lambda}= \pm \sqrt{V_{\theta}}, \\
& \Sigma \frac{d \varphi}{d \lambda}=L \sin ^{-2} \theta+a\left(\Delta^{-1} P-E\right), \\
& \Sigma \frac{d t}{d \lambda}=a\left(L-a E \sin ^{2} \theta\right)+\left(r^{2}+a^{2}\right) \Delta^{-1} P,
\end{aligned}
$$


where $\lambda=\tau / \mu, \tau$ - is a proper time of particle and

$$
\begin{aligned}
V_{r} & =P^{2}-\Delta\left[\mu^{2} r^{2}+(L-a E)^{2}+Q\right], \\
V_{\theta} & =Q-\cos ^{2} \theta\left[a^{2}\left(\mu^{2}-E^{2}\right)+L^{2} \sin ^{-2} \theta\right], \\
P & =E\left(r^{2}+a^{2}\right)+\epsilon e r-a L, \\
\Sigma & =r^{2}+a^{2} \cos ^{2} \theta \\
\Delta & =r^{2}-2 r+a^{2}+e^{2} .
\end{aligned}
$$

We will use mainly the normalized dimensionless variables and parameters: $r \Rightarrow r / M$, $a \Rightarrow a / M, e \Rightarrow e / M, \epsilon \Rightarrow \epsilon / \mu, E \Rightarrow E / \mu, L \Rightarrow L /(M \mu), Q \Rightarrow Q /\left(M^{2} \mu^{2}\right)$. The radius of BH event horizon $r=r_{+}$and the radius of $\mathrm{BH}$ inner horizon $r=r_{-}$are both the roots of equation $\Delta=0: \quad r_{ \pm}=1 \pm \sqrt{1-a^{2}-e^{2}}$.

The effective potentials $V_{r}$ and $V_{\theta}$ in (6) and (7) define the motion of particles in $r$ - and $\theta$-directions [24]. In particular, for a circular orbit at some radius $r$, equations (6) and (7) give conditions:

$$
V_{r}(r)=0, \quad V_{r}^{\prime}(r) \equiv \frac{d V_{r}}{d r}=0 .
$$

The circular orbits would be stable if $V_{r}^{\prime \prime}<0$, i. e. in the maximum of the effective potential. (Note that the effective potential $V_{r}$ is defined with the opposite sign in contrast to the usual definition of physical potential). In the case of a rotating $\mathrm{BH}$ (with $a \neq 0$ ), a particle on the orbit with $r=$ const may additionally be moving in the latitudinal $\theta$-direction. These nonequatorial orbits are called spherical orbits [25]. The purely circular orbits will correspond to the particular case of spherical orbits with parameter $Q=0$, completely confined in the $\mathrm{BH}$ equatorial plane.

The generic orbits of the third kind are nonequatorial and periodic with respect to the separate coordinates: $r, \theta$ and $\varphi$. Namely, (1) the $r$-periodicity means that the orbital radial coordinate $r$ oscillates with a period $T_{r}$ between the minimal (perigee) and maximal (apogee) values $r_{p}<r<r_{a}$. The values of $r_{p}$ and $r_{a}$ are defined by zeroes (the bounce points) of the radial potential, $V_{r}\left(r_{p, a}\right)=0$. (2) The $\theta$-periodicity means that the latitude coordinate $\theta$ oscillates with a period $T_{\theta}$ between the minimal and maximal values, $\pi / 2-\theta_{\max }<\theta<\pi / 2+\theta_{\max }$, where $\theta_{\max }$ is maximum angle of latitude elevation relative to the equatorial plane at $\theta=\pi / 2$. The value of $\theta_{\max }$ is defined by zero (the bounce point) of the latitude potential $V_{\theta}\left(\theta_{\max }\right)=0$. At last, (3) the $\varphi$-periodicity means that the azimuth coordinate $\varphi$ oscillates with a period $T_{\varphi}$ between some $\varphi_{0}$ and $\varphi_{0}+2 \pi$. These three periods are incommensurable, i.e, all ratios $T_{r} / T_{\theta} / T_{\varphi}$ are nor the rational numbers. For this reason the $3 D$ space orbit of the particle is not closed (but still periodic with respect to the separate coordinates).

\subsection{Circular orbits inside nonrotating charged BH}

We first consider the Reissner-Nordström case of nonrotating charged BH. From the joint resolution of equations (11) we find two pairs of solutions, respectively, for the 


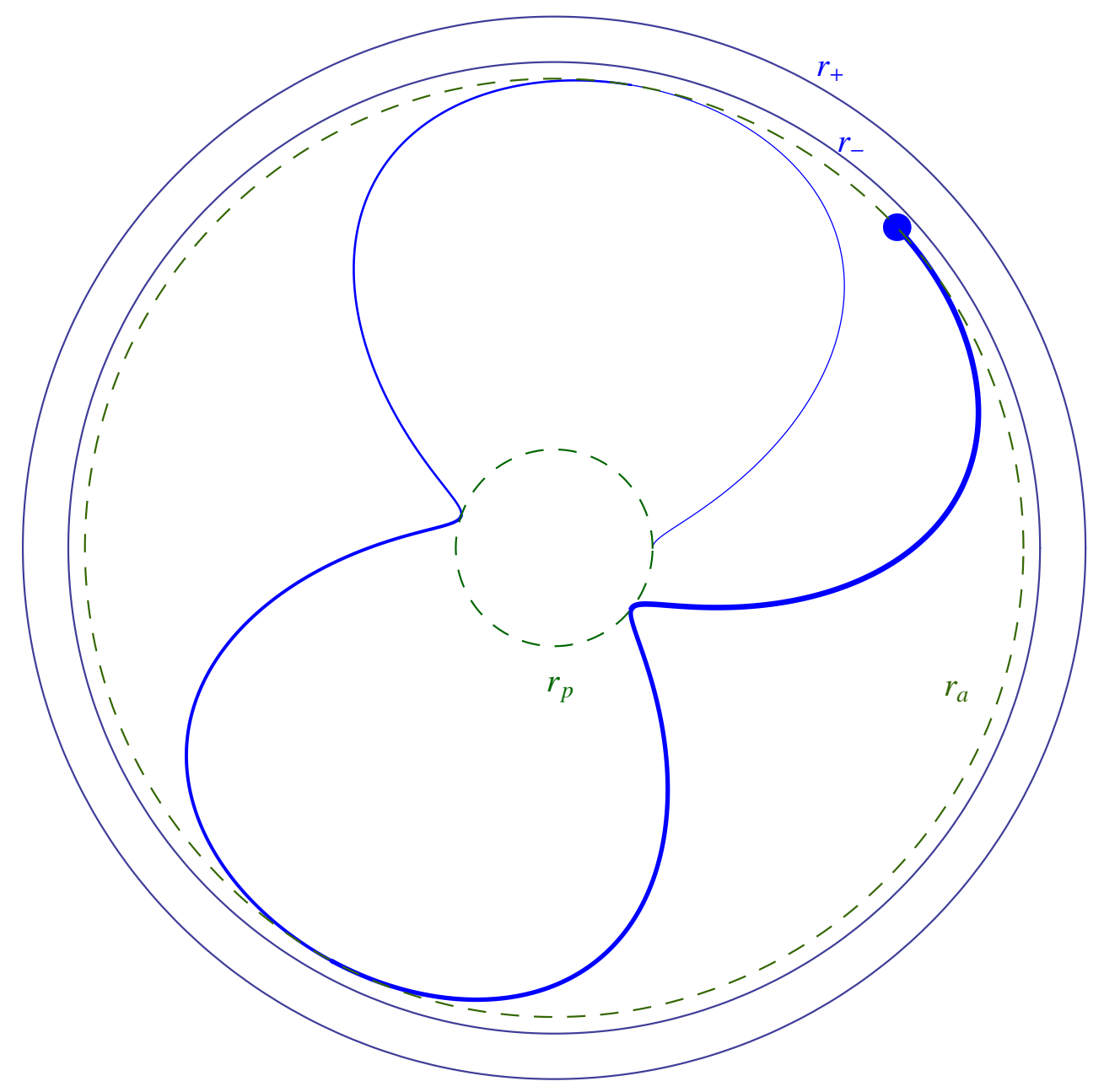

Figure 1. The stable periodic orbit of planet with a mass $\mu$ and charge $\epsilon=-1.45 \mu$ entirely inside the $\mathrm{BH}$ with a mass $M$ and charge $e=0.999 M$. Orbit parameters: $E=1.5 \mu, L=0.2 M \mu$, azimuthal and radial periods $\left(T_{\varphi}, T_{r}\right)=(14.9 M, 7.17 M)$, the perigee and apogee radii $\left(r_{p}, r_{a}\right)=(0.19 M, 0.92 M)$.

energy $E$ and angular momentum $L$ of massive particle with charge $\epsilon$ on the circular orbit with radius $r$ :

$$
\begin{aligned}
& E_{1,2}=\frac{ \pm \Delta D_{1}-e \epsilon\left(r^{2}-4 r+3 e^{2}\right)}{2 r\left(r^{2}-3 r+2 e^{2}\right)} \\
& L_{1,2}^{2}=\frac{r^{2}}{r^{2}-3 r+2 e^{2}}\left[r-e^{2}+\frac{e \epsilon \Delta\left(e \epsilon \pm D_{1}\right)}{2\left(r^{2}-3 r+2 e^{2}\right)}\right] .
\end{aligned}
$$

where

$$
D_{1}^{2}=e^{2}\left(\epsilon^{2}+8\right)+4 r(r-3) .
$$

The stability condition $V_{r}^{\prime \prime}(r, E, L)<0$ for circular orbits inside the inner horizon, $0<r<r_{-}$, is satisfied for the first pair of solution $\left(E_{1}, L_{1},\right)$ in equations (12) and (13), if $\epsilon>\mu$, and for the second pair $\left(E_{2}, L_{2},\right)$, if $\epsilon<-\mu$. Figure 1 is an example of a stable 


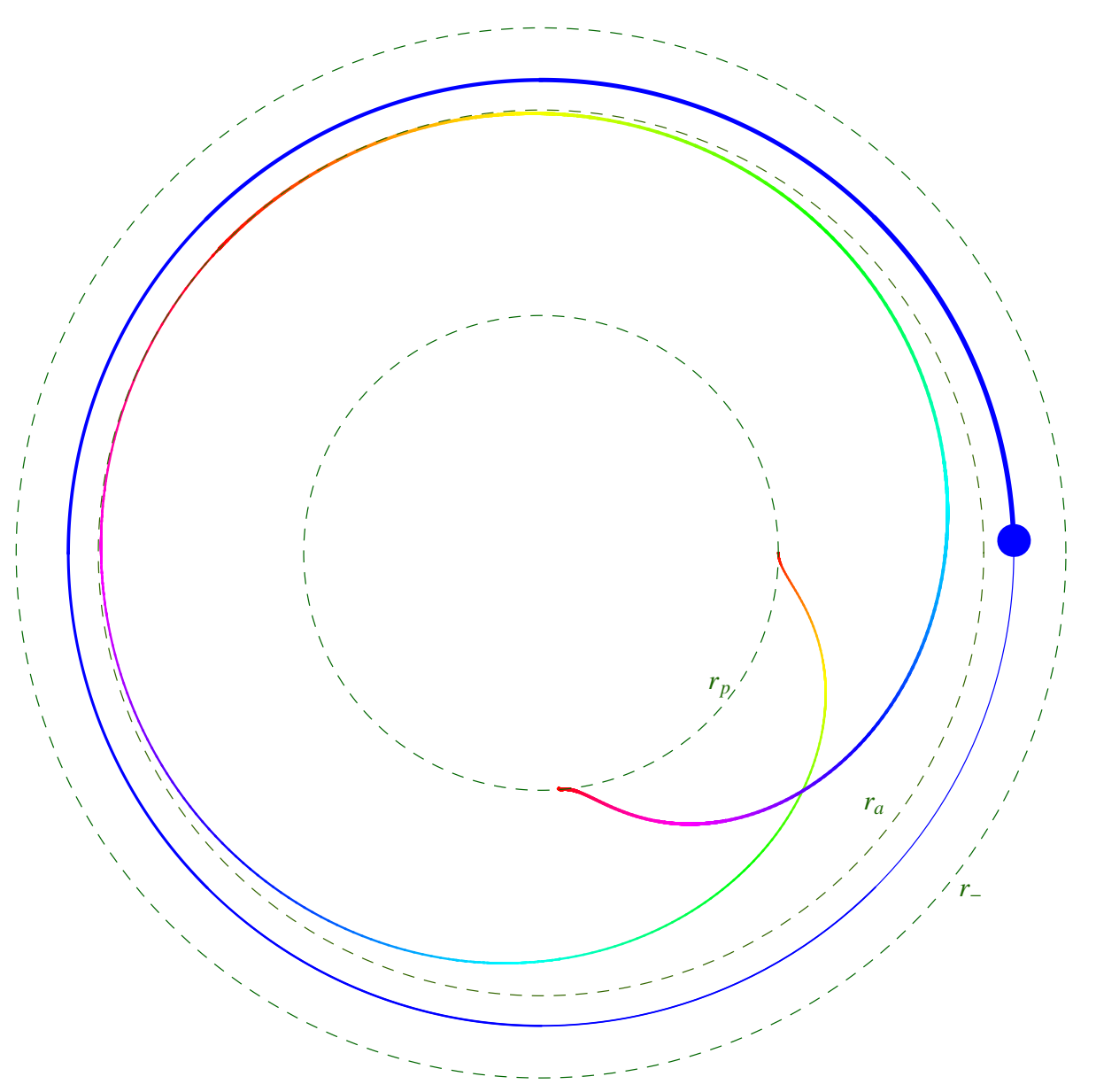

Figure 2. The stable periodic equatorial photon orbit (colored curve viewed from the north pole) with the impact parameter $b=1.53$ inside the BH with $a=0.75$ and $e=0.6$. Orbit parameters: periods $\left(T_{r}, T_{\varphi}\right)=(2.7,2.1)$, perigee and apogee (dashed circles) $\left(r_{p}, r_{a}\right)=(0.33,0.61)$. The external dashed circle is the inner horizon with $r=r_{-}=0.72$. The blue circle is the circular planet orbit with the radius $r=0.65$, energy $E=10.5$ and impact parameter $b=1.54$. The angular momentum of the black hole is directed to the north pole. The thicknesses of orbital curves are growing with time. Note that the equatorial photon and planet, starting at the azimuth angle $\varphi=0$, are both orbiting in opposite direction with respect to $\mathrm{BH}$ rotation.

periodic orbit of a charged particle inside a nonrotating $\mathrm{BH}$, calculated numerically from equations (2) and (4), using a general formalism for motion in the central field [26]. The standard finite difference method was used in numerical integrations of equations (2), (3) and (4) for the noncircular orbits with the finite displacements of a proper time $\Delta \tau$ as the integration steps (or by using the corresponding finite displacements of affine parameter $\lambda$ along the photon orbit). 


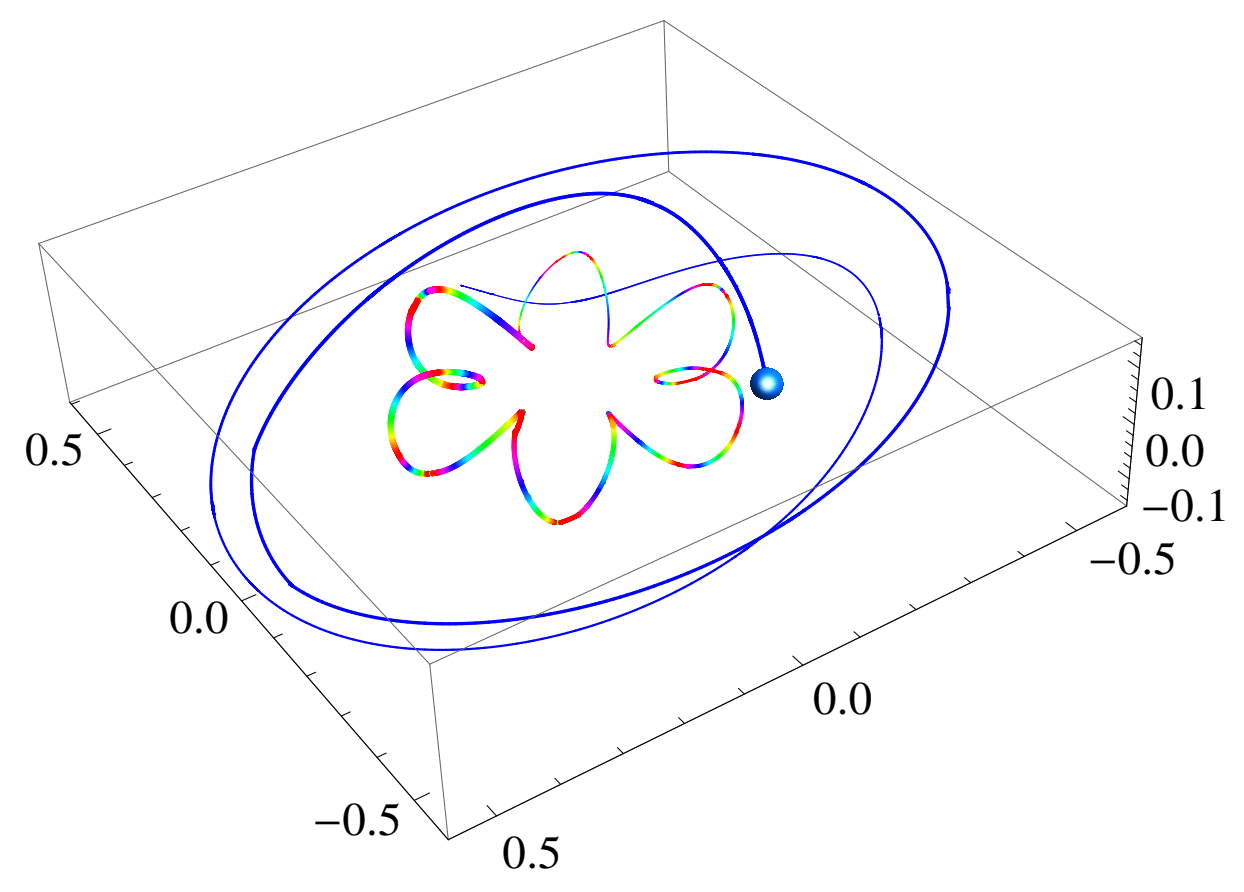

Figure 3. Outer curve: the stable periodic orbit of planet with orbital parameters $(E, L, Q)=(0.568,1.13,0.13)$, periods $\left(T_{\varphi}, T_{r}, T_{\theta}\right)=(1.63,3.70,1.17)$, apogee and perigee radii $\left(r_{p}, r_{a}\right)=(0.32,0.59)$ and the maximum angle of latitude elevation relative to the equatorial plane $\theta_{\max }=14.6^{\circ}$ inside the $\mathrm{BH}$ with $a=0.9982$ and $e=0.05$. Inner curve: the stable periodic nonequatorial photon orbit with orbit parameters: $(b, q)=(1.38,0.03),\left(T_{\varphi}, T_{r}, T_{\theta}\right)=(2.95,0.49,0.33),\left(r_{p}, r_{a}\right)=(0.14,0.29)$ and $\theta_{\max }=10.1^{\circ}$ inside the same $\mathrm{BH}$. The starting parts of orbits are thin, while the ending parts are thick

\subsection{Spherical orbits of neutral particles inside $B H$}

In the Kerr-Newman case the stable circular orbits (and also the stable spherical ones) exist inside the inner horizon not only for charged particles, but also for neutral ones $(\epsilon=0)$ and photons $(\mu=0)$. From equations (11) we find two pairs of solutions for energy $E$ and azimuthal impact parameter $b=L / E$ of neutral massive particle on the spherical orbit:

$$
\begin{aligned}
& E_{1,2}^{2}=\frac{\mp 2 D_{2}+\beta_{1} r^{2}+a^{2}\left[2\left(r-e^{2}\right) \Delta-r^{2}(r-1)^{2}\right] Q}{r^{4}\left[\left(r^{2}-3 r+2 e^{2}\right)^{2}-4 a^{2}\left(r-e^{2}\right)\right]} \\
& b_{1,2}=\frac{ \pm D_{2} r-a^{2}\left(r-e^{2}\right)\left\{\beta_{2} r+\left[a^{2}-r\left(r-e^{2}\right)\right] Q\right\}}{a\left(r-e^{2}\right)\left\{r\left[\left(\Delta-a^{2}\right)^{2}-a^{2}\left(r-e^{2}\right)\right]+a^{2}(1-r) Q\right\}},
\end{aligned}
$$

where

$$
\begin{aligned}
& \beta_{1}=\left(r^{2}-3 r+2 e^{2}\right)\left(r^{2}-2 r+e^{2}\right)^{2}-a^{2}\left(r-e^{2}\right)\left[r(3 r-5)+2 e^{2}\right], \\
& \beta_{2}=e^{4}-a^{2}\left(r-e^{2}\right)+2 e^{2} r(r-2)-r^{2}(3 r-4), \\
& D_{2}^{2}=\left[a\left(r-e^{2}\right) \Delta\right]^{2}\left[\left(r-e^{2}\right) r^{4}-r^{2}\left(r^{2}-3 r+2 e^{2}\right) Q+a^{2} Q^{2}\right] .
\end{aligned}
$$




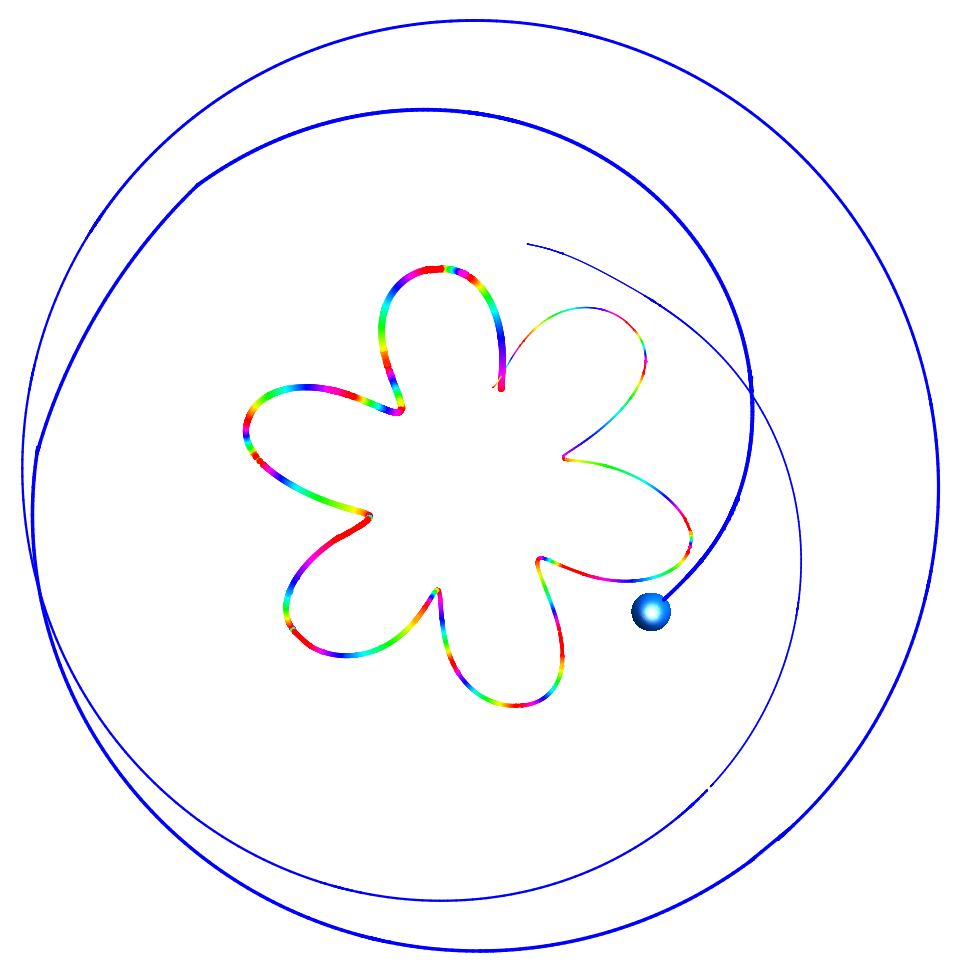

Figure 4. The stable periodic orbits of photon and planet (shown in figure 3) viewed from the coordinate frame north pole. The naked central singularity is glowing in the center. The starting parts of orbits are thin and the finishing parts are thick.

It can be shown that stable spherical orbits are realized for the first pair of solution $\left(E_{1}, b_{1}\right)$ with $0<Q<Q_{\max }$, where $Q_{\max }$ is a root of the marginal stability equation $V_{r}^{\prime \prime}=0$. All spherical orbits with $Q<0$ are unstable (see also [25]). The analogous formulae for spherical orbits of charged particles in the Kerr-Newman case are very cumbersome, and we are not reproducing them here.

\subsection{Spherical orbits of photons inside $B H$}

The spherical photon orbit corresponds to the ultrarelativistic limit for massive particle energy on the spherical orbit, $E \rightarrow \infty$. This limit is equivalent to the case $\mu=0$. Photon orbit depends on two parameters, the azimuthal impact parameter $b=L / E$ and the latitudinal (tangential) impact parameter $q=Q / E^{2}$. Equations (15) in the ultrarelativistic limit are very simplified:

$$
\begin{aligned}
& b_{1}=\frac{\left.a^{2}(1+r)+r\left(r^{2}-3 r+2 e^{2}\right)\right]}{a(1-r)}, \quad b_{2}=\frac{a^{2}+r^{2}}{a}, \\
& q_{1}=\frac{r^{2}\left[4 a^{2}\left(r-e^{2}\right)-\left(r^{2}-3 r+2 e^{2}\right)^{2}\right]}{a^{2}(1-r)^{2}}, \quad q_{2}=-\frac{r^{4}}{a^{2}} .
\end{aligned}
$$

Here the first pair of impact parameters, $\left(b_{1}, q_{1}\right)$, corresponds to stable spherical photon orbits, while the second pair, $\left(b_{2}, q_{2}\right)$, to unstable ones. The stability condition $V_{r}^{\prime \prime} \leq 0$ 


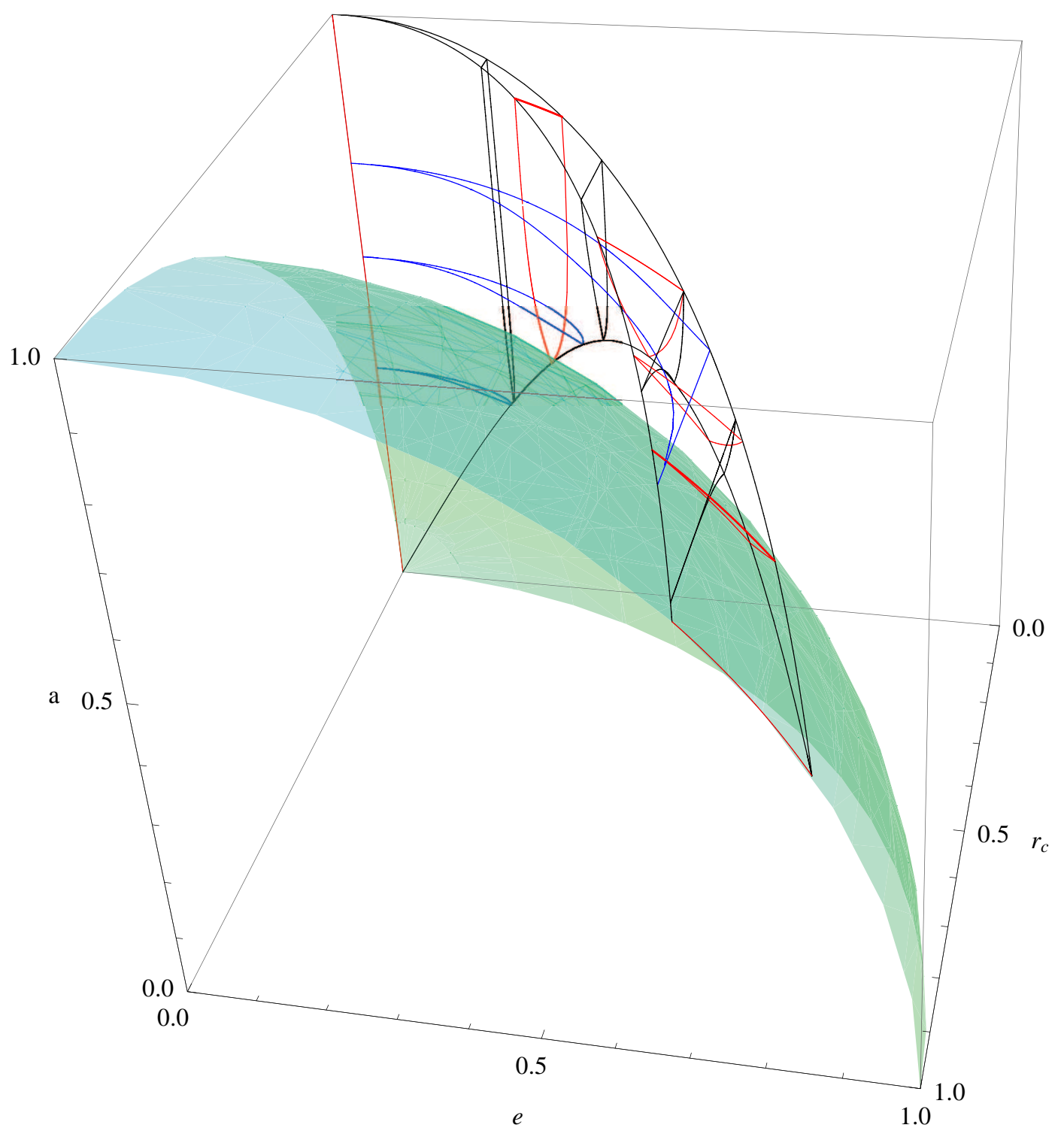

Figure 5. $3 D$ domain of existence (marked by the level curves $r=$ const, $a=$ const, $e=$ const) for the stable circular (equatorial) orbits of neutral particles inside the inner $\mathrm{BH}$ horizon (a filled part of sphere). The rear filled surface corresponds to the ultrarelativistic limit or circular photon orbits. The stable spherical (nonequatorial) orbits for massive particle exist at radii, limited by the rear surface of circular photons and the inner horizon.

for spherical photons with the first pair of impact parameters $\left(b_{1}, q_{1}\right)$ is written in the form

$$
a^{2}+e^{2}-r\left(r^{2}-3 r+3\right) \leq 0
$$

From this inequality we find the upper limit of impact parameter $q_{1}>0$ for stable spherical photons:

$$
q_{1} \leq\left(\frac{1-\delta^{1 / 3}}{a}\right)^{2}\left[3-4 e^{2}-2\left(3-2 e^{2}\right) \delta^{1 / 3}+3 \delta^{2 / 3}\right],
$$


where $\delta=1-a^{2}-e^{2}$. The maximal allowable value for $q_{1}$ is reached for the extreme BH with $a=\sqrt{1-e^{2}} \leq 1 / 2, e \leq \sqrt{3} / 2$ :

$$
q_{1, \max }=4-a^{-2} \leq 3 .
$$

Figure 2 is an example of stable periodic equatorial photon orbit inside the slightly charged but near extremely rotating BH. Figures 3 and 4 are, respectively, the examples of stable periodic planet and photon orbits inside a slightly charged but near extremely rotating $\mathrm{BH}$ with the canonical specific angular momentum $a_{\mathrm{lim}}=0.09982$ due to untwisting by a thin accretion disk [27].

\subsection{Circular orbits of photons inside $B H$}

The spherical orbits become circular ones in the particular case of $Q=0$. A corresponding relation for circular photon orbits follows in the relativistic limit from equation (15) or from (19) and (20):

$$
4 a^{2}\left(r-e^{2}\right)=\left(r^{2}-3 r+2 e^{2}\right)^{2} .
$$

with two possible solutions for impact parameter

$$
b_{1,2}=\frac{a \beta_{2} \pm r^{2} \sqrt{\left(r-e^{2}\right) \Delta^{2}}}{\left(r^{2}-2 r+e^{2}\right)^{2}-a^{2}\left(r-e^{2}\right)},
$$

where $\Delta$ and $\beta_{2}$ are, respectively, from (10) and (17). In (25) the first solution $q_{1}$ (with a plus sign) corresponds to the stable orbit, whereas $q_{2}$ corresponds to the unstable one. See in figure 5 the $3 D$ domain of existence for the stable circular photons inside $\mathrm{BH}$. These orbits exist at $e^{2} \leq r \leq(4 / 3) e^{2}, a \neq 0,0<e \leq \sqrt{3} / 2$ and $0<b<5 / 2$. Note, that equatorial stable orbits for planets and photons inside the Kerr $\mathrm{BH}(e=0)$ are absent at all. The third kind orbits inside the Kerr BH are only nonequatorial ones.

\section{Conclusions}

Inside the inner Cauchy horizon of the Kerr-Newman BH there are stable periodic orbits of particles (planets) and photons (orbits of the third kind). In the case of a nonrotating charged $\mathrm{BH}(a=0)$ the stable periodic orbits exist only for particles with a large enough charge. All the stable periodic planet and photon orbits inside the rotating and noncharged $\mathrm{BH}(e=0)$ are nonequatorial. We hypothesize that civilizations of the third type (according to Kardashev scale [28]) may live safely inside the supermassive $\mathrm{BHs}$ in the galactic nuclei being invisible from the outside. Some additional highlighting during the night time comes from eternally circulating photons. Yet, some difficulties (or advantages?) of a life inside BH are worth mentioning, such as a possible causality violation [8, 9, 10] and the growing energy density and mass inflation in the close vicinity of the Cauchy horizon [12, 13, 14, 15, 16]. The existence of third kind orbits inside the event horizon may be verified or falsified in principle (without the traveling into black holes) by the future observations of white holes. 


\section{Acknowledgments}

Author acknowledges E. O. Babichev, V. A. Berezin, Yu. N. Eroshenko and I. D. Novikov

for fruitful discussions. This work was supported in part by the Russian Foundation for Basic Research grant 10-02-00635.

\section{References}

[1] Misner C W, Thorne K S and Wheeler J A 1973 Gravitation (W. H. Freeman and Company, San Francisco) Chapter 34

[2] Chandrasekhar S 1983 The mathematical theory of black holes (Oxford University Press, Oxford) Chapters 5 and 7

[3] Wald R M 1984 General Relativity (The University of Chicago Press, Chicago 60673)

[4] Frolov V P and Novikov I D 1998 Black hole physics: basic concepts and new developments (Dordrecht: Kluwer Academic) Chapters 6 and 14

[5] Frolov V P, Markov M A and Mukhanov V F 1989 Phys. Lett. B 216272

[6] Frolov V P, Markov M A and Mukhanov V F 1990 Phys. Rev. D 41383

[7] Barrabes C and Frolov V P 1996 Phys. Rev. D 533215

[8] Carter B 1966 Phys. Rev. 1411242

[9] Carter B 1966 Phys. Lett. 21423

[10] Carter B 1968 Phys. Rev. 1741550

[11] Zeldovich Ya B and Novikov I D 1971 Relativistic astrophysics Vol 1: Stars and relativity (Chicago: University of Chicago Press) Chapter 3

[12] Simpson M and Penrose R 1973 Intern. J. Theor. Phys. 7183

[13] Gursel Y, Sandnerg V D, Novikov I D and Starobinsky A A 1979 Phys. Rev. D 19413

[14] Gursel Y, Sandnerg V D, Novikov I D and Starobinsky A A 1979 Phys. Rev. D 201260

[15] Novikov I D and Starobinsky A A 1980 Sov. Phys. - JETP 511

[16] Chandrasekhar S and Hartle J B 1982 Proc. Roy. Soc. Ser. A 384301

[17] Dotti G, Gleiser R J, Ranea-Sandoval I F and Vucetich H 2008 Class. Quant. Grav. 25245012

[18] Bičák J, Stuchlík Z and Balek V 1989 Bull. Astron. Inst. Czechosl. 4065

[19] Balek V, Bičák J and Stuchlík Z 1989 Bull. Astron. Inst. Czechosl. 40133

[20] Grunau S and Kagramanova V 2011 Geodesics of electrically and magnetically charged test particles in the Reissner-Nordstro"m space-time: analytical solutions Preprint arXiv:1011.5399 [gr-qc]

[21] Olivares M, Saavedra J, Leiva C and Villanueva J R 2011 Motion of charged particles on the Reissner-Nordstro"m (Anti)-de Sitter black holes Preprint arXiv:1101.0748 [gr-qc]

[22] Hackmann E, Kagramanova V, Kunz J and Lam̈merzahl C 2010 Phys. Rev. D 81044020

[23] Pugliese D, Quevedo H and Ruffini R 2010 Circular motion of neutral test particles in ReissnerNordstrom spacetime Preprint arXiv:1012.5411 [astro-ph.HE]

[24] Bardeen J M, Press W H and Teukolsky S A 1972 Astrophys. J. 178347

[25] Wilkins D C 1972 Phys. Rev. D 5814

[26] Landau L D and Lifshitz E M 1969 Mechanics, Course of theoretical physics (Oxford: Pergamon Press, 2nd ed.) Chapter III

[27] Thorne K S 1974 Astrophys. J. 191507

[28] Kardashev N S 1964 Sov. Astron. - AJ 8, 217 